\title{
In vivo toxicity of quantum dots: no cause for
}

\section{concern?}

\author{
"Naturally, substantial time and resources will be required to perfect and \\ standardize quantum dot formulations ... We should not give up hope even if this \\ process seems slow."
}

\section{KEYWORDS: nanocrystal $\approx$ quantum dot $₫$ toxicity}

Semiconductor nanocrystals, also known as quantum dots (QDs), possess unique optical properties that make them useful as fluorescent probes or traceable nanocarriers for in vivo applications ranging from imaging to theranostics [1]. The surfaces of QDs can be conjugated with biomolecules to enable in vivo targeted imaging and drug delivery $[2,3]$. These unique capabilities and qualities of QDs have made them a powerful platform that can help to reveal important biological insights. Ultimately, they may also provide unique benefits in clinical diagnostic and therapeutic applications [4]. However, progress toward clinical applications has been delayed by concerns about the potential toxicity of QDs. Much of the QDs community has been hesitant to work toward clinical applications, based on reports demonstrating release of toxic heavy metal ions from degradation of QDs in cell culture studies [5]. In addition, photoexcited QDs have been shown to generate reactive oxygen species that are highly toxic to cells [6]. On the other hand, in small animal studies, bioconjugated QDs did not have any observable ill effects at concentrations appropriate for in vivo imaging applications [7-9]. Thus, conclusions drawn from in vitro and in vivo studies remain somewhat contradictory and do not yet provide a sound basis for confident prediction of in vivo toxicity in humans.

\section{Factors influencing QD toxicity in vivo}

Many factors influence the potential toxicity of QDs, through different biological interactions in vivo. Along with the local cytotoxicity associated with heavy-metal release and generation of reactive oxygen species that can be observed in vitro, QDs may interact directly with blood components. They may accumulate nonspecifically in high-blood-flow organs, and they may induce immune responses [10]. Each of these interactions depends on the size and surface chemistry of the QD formulation in different ways. In small animal studies, intravenously administered QDs mainly accumulated in the liver, spleen and lymphatic system; very little accumulation was observed in the heart, lungs and kidneys [7]. However, the biodistribution was also shown to vary for different QD formulations [11]. For bioconjugated QDs that do not aggregate in vivo, the excretion rate depends most strongly on the hydrodynamic diameter of the QDs [12]. One strategy for reducing QD toxicity is therefore to minimize the hydrodynamic diameter to a point where rapid renal excretion occurs [13]. While the diameter of inorganic QD cores is typically $2-5 \mathrm{~nm}$, the hydrodynamic diameter of QD formulations can vary widely, from individual QDs with direct bioconjugation and hydrodynamic diameter below $10 \mathrm{~nm}$ to polymer micelles encapsulating multiple core-shell QDs with hydrodynamic diameter up to $200 \mathrm{~nm}$.

Some toxicity concerns may be alleviated by changes in the composition of the inorganic QD core. For example, local cytotoxicity related to release of $\mathrm{Cd}$ ions can be eliminated by using QD formulations based upon indium phosphide (InP) or silicon [14-16]. InP-based core-shell QDs are approaching the performance levels of the better-studied Cd-based QDs, and may ultimately achieve the same desirable optical properties. However, while $\mathrm{InP}$ is free of $\mathrm{Cd}$, In is also a heavy metal with no known biological role, and its presence may be a cause for concern. Silicon QDs are expected to be more biocompatible; they should ultimately degrade to silicic acid, which can be excreted in the urine. In vivo bioimaging using silicon QDs has been demonstrated $[14,15]$. However, for the foreseeable future, the optical properties of silicon QDs are unlikely to

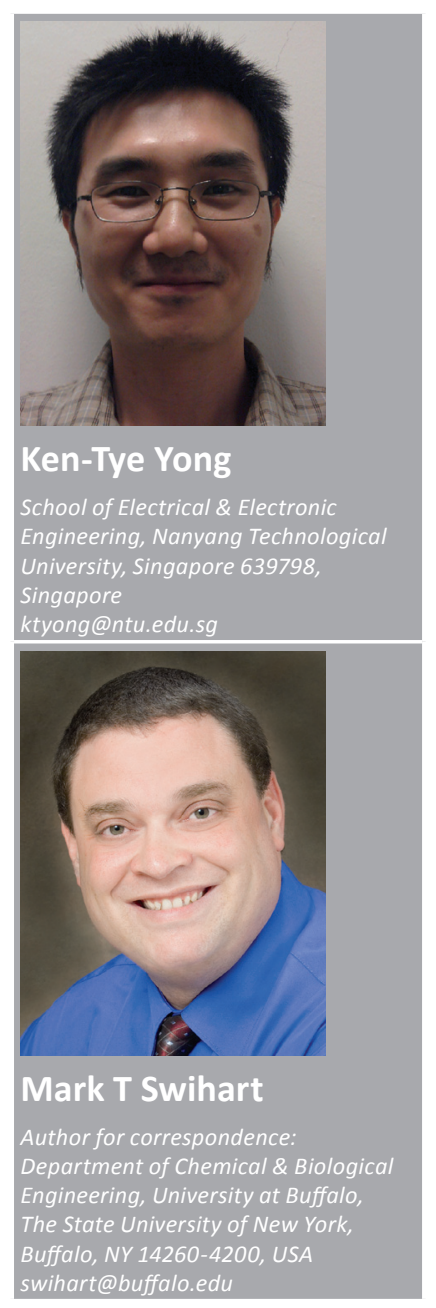

\section{Future $\mathrm{fSS}$}


match the Cd-based formulations. Other toxicity mechanisms, such as photogeneration of reactive oxygen species, are more general to all QDs $[6,17]$. Biodistribution and interactions, such as induced immune responses, are expected to be unaffected by the QD core composition. Thus, while some alternate formulations are available or emerging, Cd-based QDs will remain of interest for some time, and in vivo studies of them are likely to produce insights that are also relevant to formulations with different QD core materials.

"...while some alternate formulations are available or emerging, Cd-based quantum dots will remain of interest for some time..."

Present understanding of the toxicological and pharmacological effects of QDs remains rather poor [18]. This lack of understanding is largely a result of the variety of QD formulations considered in the literature combined with the variety of toxicity assays, animal models and dosage measures used. To date, more than 70 types of bioconjugated QD formulations have been reported. Many of these have been tested in vitro but not in vivo. With such limited information and so many formulations, judging whether a specific QD formulation is suitable for in vivo use remains a major challenge for the QD research community.

\section{Future needs \& current directions}

A comprehensive study of the toxicity of a few standardized QD formulations, including longterm toxicological and pharmacokinetic investigations of the degradation, excretion, persistence and immunogenicity of QDs, will ultimately be needed if QD formulations are to reach the clinic in applications such as image-guided surgery for tumor removal. However, such studies are tedious, complex and, by definition, long. These in vivo toxicity experiments are expensive to perform and require the long-term care of small animals by trained professionals. In an environment of limited research resources, such studies must be coordinated and standardized to minimize unnecessary duplication and to ensure that results for different QD formulations can be reliably compared.

\section{"A comprehensive study of the toxicity of a few standardized quantum dot formulations ... will ultimately be needed if quantum dot formulations are to reach the clinic..."}

The current literature is showing an increasing trend for researchers from different countries and scientific backgrounds to collaborate to investigate the long-term toxicity of QDs in vivo, using animals ranging from mice to primates. This is an encouraging step toward optimally sharing and utilizing 'global resources' for evaluating the long-term toxicity of QDs in vivo. For example, our groups in the physical science and engineering departments in the USA and Singapore have recently collaborated with medical researchers at a hospital in China to investigate the in vivo toxicity of QDs in nonhuman primates [19]. The results were encouraging, showing that rhesus macaques injected with phospholipid micelleencapsulated CdSe/CdS/ZnS QDs at relatively high dosage did not exhibit any evidence of acute toxicity. All blood chemistry and biochemical markers remained within normal ranges over a 90-day period following intravenous injection of the QD formulation and histology of major organs did not show any abnormalities 90 days after injection. This study serves to temper some of the concerns over the toxicity of QDs intended for clinical applications. Nevertheless, chemical analysis showed that the vast majority of the initial QD dose remained in the liver, spleen and kidneys of the animals 90 days after injection. This suggests that the degradation and excretion of QDs in this formulation is quite slow. Longer-term studies will be needed to investigate the ultimate distribution of these QDs and the impact of their persistence in primates.

\section{A broader perspective}

Some useful perspectives on the toxicity of QDs can be provided by comparison with other toxic yet clinically useful formulations. Consider paclitaxel, a common chemotherapy drug used worldwide [20]. The drug was discovered in 1967 when this compound was isolated from the Pacific yew tree. Paclitaxel, a mitotic inhibitor that is used to treat a large variety of cancers, was first employed to treat both ovarian cancer and melanoma cases in 1988. Its side effects include nausea and vomiting, loss of appetite, change in taste, thinned or brittle hair, pain in the joints of the arms or legs, changes in the color of the nails and tingling in the hands or toes. Bone marrow suppression, mucositis and peripheral neuropathy occur at higher doses (beyond the clinical range). Nonetheless, many patients are successfully treated and cured each year with paclitaxel, at appropriate dosages and following appropriate treatment regimens. It took over 35 years to optimize the paclitaxel formulation and dosage that is used clinically today and the search for better targeted formulations with fewer side effects 
continues. Paclitaxel is widely used because its benefits outweigh the associated risks. QD formulations for in vivo biomedical applications are in a position somewhat similar to that of paclitaxel 30 years ago. For applications such as sentinel lymph node mapping or imaging-guided surgery using antibody-targeted QDs to label cancerous tissue, the benefits of QDs as imaging agents may dramatically outweigh the risks associated with their use. Naturally, substantial time and resources will be required to perfect and standardize QD formulations for specific applications to a level where they can be tested in clinical trials. We should not give up hope even if this process seems slow.

\section{"To most efficiently move toward clinical applications, we must communicate and coordinate activities across both geographical and disciplinary boundaries."}

The QD research community today includes many active and distinguished researchers worldwide who are engineering and testing QDs that will eventually lead to formulations that are employed clinically for human diagnostic and therapeutic applications. These researchers come from diverse backgrounds ranging from physical chemistry and materials science to molecular biology and clinical medicine. To most efficiently move toward clinical applications, we must communicate and coordinate activities across both geographical and disciplinary boundaries. In response to the question in the title of this article: yes, there is still much cause for concern about the in vivo toxicity of QDs, but there is also much cause for hope as researchers cross boundaries and work together to identify and address toxicity concerns and demonstrate applications where the benefits of QD use outweigh the associated risks.

\section{Financial \& competing interests disclosure}

The authors' research activities in the area of quantum dots for biological applications have been supported by the US NIH, The John R Oishei Foundation, The University at Buffalo (SUNY), Nanyang Technological University and the Singapore Ministry of Education. The authors have no other relevant affiliations or financial involvement with any organization or entity with a financial interest in or financial conflict with the subject matter or materials discussed in the manuscript.

No writing assistance was utilized in the production of this manuscript.

\section{References}

1 Prasad PN. Nanomedicine: Transforming Healthcare with Nanotechnology. John Wiley \& Sons, Hoboken, NJ, USA (2012).

2 Biju V, Itoh T, Ishikawa M. Delivering quantum dots to cells: bioconjugated quantum dots for targeted and nonspecific extracellular and intracellular imaging. Chem. Soc. Rev. 39(8), 3031-3056 (2010).

3 Zrazhevskiy P, Sena M, Gao X. Designing multifunctional quantum dots for bioimaging, detection, and drug delivery. Chem. Soc. Rev. 39 (11), 4326-4354 (2010).

4 Taylor A, Wilson KM, Murray P, Fernig DG, Levy R. Long-term tracking of cells using inorganic nanoparticles as contrast agents: are we there yet? Chem. Soc. Rev. 41(7), 2707-2717 (2012).

5 Derfus AM, Chan WCW, Bhatia SN. Probing the cytotoxicity of semiconductor quantum dots. Nano Lett. 4(1), 11-18 (2003).

6 Ipe BI, Lehnig M, Niemeyer CM. On the generation of free radical species from quantum dots. Small 1(7), 706-709 (2005).

7 Hauck TS, Anderson RE, Fischer HC, Newbigging S, Chan WCW. In vivo quantum-dot toxicity assessment. Small 6(1), 138-144 (2010).
8 Yong K-T, Roy I, Law W-C, Hu R. Synthesis of cRGD-peptide conjugated near-infrared $\mathrm{CdTe} / \mathrm{ZnSe}$ core-shell quantum dots for in vivo cancer targeting and imaging. Chem. Commun. 46(38), 7136-7138 (2010).

9 Bruns OT, Ittrich $\mathrm{H}$, Peldschus K et al. Real-time magnetic resonance imaging and quantification of lipoprotein metabolism in vivo using nanocrystals. Nat. Nanotechnol. 4(3), 193-201 (2009).

10 Dobrovolskaia MA, McNeil SE. Immunological properties of engineered nanomaterials. Nat. Nanotechnol. 2(8), 469-478 (2007).

11 Schipper ML, Iyer G, Koh AL et al. Particle size, surface coating, and PEGylation influence the biodistribution of quantum dots in living mice. Small 5(1), 126-134 (2009).

12 Choi SH, Liu W, Misra P et al. Renal clearance of quantum dots. Nat. Biotechnol. 25(10), 1165-1170 (2007).

13 Choi HS, Liu W, Liu F et al. Design considerations for tumour-targeted nanoparticles. Nat. Nanotechnol. 5(1), 42-47 (2009).

14 Erogbogbo F, Yong K-T, Roy I et al. In vivo targeted cancer imaging, sentinel lymph node mapping and multi-channel imaging with biocompatible silicon nanocrystals. ACS Nano 5(1), 413-423 (2010).

15 Park J-H, Gu L, Von Maltzahn G, Ruoslahti E, Bhatia SN, Sailor MJ. Biodegradable luminescent porous silicon nanoparticles for in vivo applications. Nat. Mater. 8(4), 331-336 (2009).

16 Yong K-T, Ding H, Roy I et al. Imaging pancreatic cancer using bioconjugated InP quantum dots. ACS Nano 3(3), 502-510 (2009).

17 Green M, Howman E. Semiconductor quantum dots and free radical induced DNA nicking. Chem. Commun. (1), 121-123 (2005).

18 Chou LY, Chan WC. Nanotoxicology: no signs of illness. Nat. Nanotechnol. 7(7), 416-417 (2012).

19 Ye L, Yong K-T, Liu L et al. A pilot study in non-human primates shows no adverse response to intravenous injection of quantum dots. Nat. Nanotechnol. 7(7), 453-458 (2012).

20 Rowinsky EK, Donehower RC. Paclitaxel (Taxol). N. Engl. J. Med. 332(15), 1004-1014 (1995). 